\title{
Development of an integrated system for distribution planning in supply chain
}

\author{
A. Modares* and M. Sepehri \\ Graduate School of Management and Economics \\ Sharif University of Technology, Tehran, Iran \\ modares@sharif.edu
}

Received March 2009

\begin{abstract}
Distribution planning, which includes Vehicle Routing and Scheduling Problem (VRSP), has become an important element in Supply Chain impacting its service level and efficiency. Computer Aided Routing and Scheduling (CARS) has been developed and implemented, which can handle complicated distribution models using advanced heuristic optimization algorithms. A classification scheme is introduced to classify various types of routing and scheduling problems in a structured manner, based on the main objects of VRSP. The integrated system described in this paper can manage the dynamic aspects of the Supply Chain in practice. The modelling and solution approach in the CARS optimization engine, its user interface, sample performance measurements, and planning and operational features of the system are described in detail.
\end{abstract}

*To whom all correspondence should be addressed.

\section{Introduction}

Distribution plays an essential role in the delivery of value within the supply chain, since value is not realized until the product reaches where it is needed. With the growth of ecommerce and home delivery services, distribution costs have become more important. Widespread application of outsourcing strategies, which has reshaped organizations from centralized manufacturing facilities to geographically dispersed networks of resources, also increases the importance of distribution services (Chopra, Dougan \& Taylor, 2001; Chopra \& Miegham, 2000).

The most important planning and operational decision related to distribution planning in the supply chain planning is the routing and scheduling of deliveries (Chopra et al., 2001). Vehicle Routing and Scheduling Problem (VRSP) is a significant issue in both supply chain planning and distribution optimization (Beasley, 1983; Bodin et al., 1983).

The primary objective of this paper is to introduce and describe a distribution planning system, based on an innovative VRSP classification scheme. The general user should appreciate the simplicity of the application and at the same time the multiple features and integration of the system. This information is believed to enhance the knowledge of system developers and operations managers in developing and evaluating optimization systems for distribution planning in Supply Chain. The system contains innovative algorithms and an advanced optimization engine. However, the primary attention here is paid to the managerial issues and assuring the applicability of the system.
The reminder of this paper is organized as follows. After describing the development motivation, a classification scheme for VRSP will be given in the following section. The modelling and solution approach for solving real routing problems will be explained in Section 4. The optimization algorithms and implementation results will be presented in Section 5 and 6, respectively. Capabilities and important features of CARS will be discussed in Section 7. Finally, management aspects of the application, some concluding remarks and the direction for further research will be done in Section 8 .

\section{Development motivation}

Companies are building responsive supply chains and seeking an efficient resource utilization to face off stiff competition and customer pressure for higher service levels. A critical decision relates to distribution planning and in particular to VSRP. A large body of research and modelling literature has been devoted to VRSP (Chopra \& Meindl, 2004). Most of such work, however, concentrated on hypothetical or simplified problems and disregarded many practical aspects of such problems. At the same time, many practical problems have been tackled by different commercial systems, but little has been published about them.

Computer Aided Routing and Scheduling (CARS) is a distribution planning system developed to handle various types of problems arising in practice. CARS has avoided the typical commercial software issues, such as using suboptimum solutions, non-user friendly and complex interface applications, or static optimization. In a handful of practice applications to date, CARS has yielded significant improvements over traditional systems or intuitive solutions. 
CARS may be efficiently customized to meet a wide range of application characteristics and users' needs. Its optimization algorithm is general enough to cover a range of dimensions and parameters. Since the system development was based on a comprehensive review of various types of practical situations, it is expected to become popular in industries with complicated supply chain requirements. Important features of CARS may be stated as follows:

- High flexibility to meet various logistics situations and clients needs.

- Powerful optimization algorithm to tackle complex problems effectively.

- Intuitive graphical user interface for effective analysis and improvement of results.

- Flexibility to integrate the users know how with the computational power of advanced optimization algorithms.

\section{VRSP classification}

A wide variety of variables in VRSP makes the system complex, and thus calls for an appropriate classification scheme (Derochers, Lenstra \& Savelsberh, 1990). The proposed system should handle various types of distribution planning cases. Such complex cases can only be modeled by proper definition of the attributes of VRSP objects. Figure 1 illustrates a typical distribution network.

\section{Figure 1: A simplified distribution network}

We define VRSP simply as "planning the efficient flow of goods between facilities by a fleet of carriers through the distribution networks'. This statement reveals the main components or objects of VRSP problems, namely, goods, facilities, carriers and distribution networks. Each object may have its owners, who impose their objectives and restrictions on the problem. For example, drivers may be considered as the owners of vehicles who may impose working time or region constraints. The distribution planning model is depicted in Figure 2.

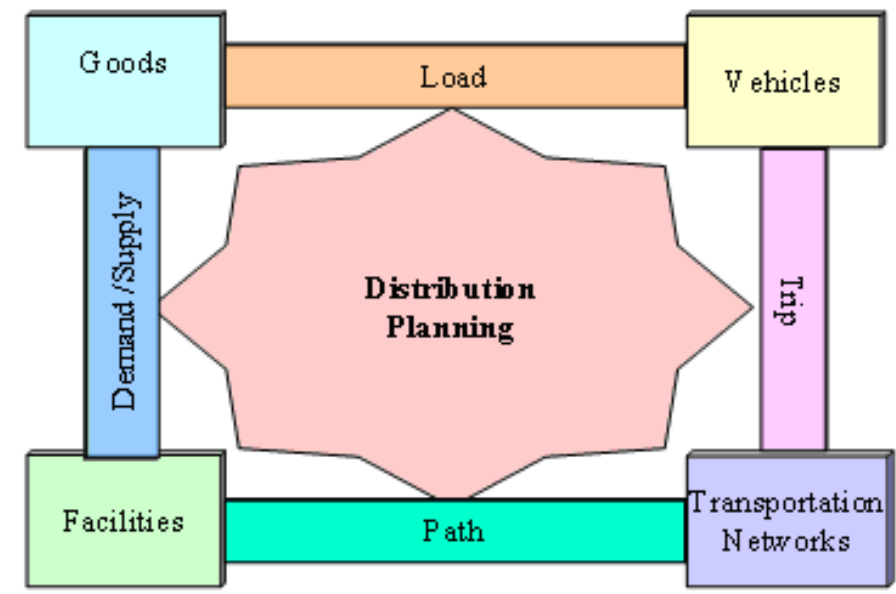

Figure 2: The distribution planning model

In this section, a classification framework is developed based on main components of VRSP. The system consists of objects with interrelated attributes. Objects are related to each other through links, as in Figure 2, described below.

- $\quad$ Load, the link between goods and vehicles, is a set of products or parts which are put on vehicles.

- Trip, the link between vehicles and distribution networks, is a set of paths that a vehicle should traverse.

- Path, the link between distribution networks and facilities, is the set of arcs that connects locations.

- Demand or supply, the link between facilities and goods, is a collection of goods that should be transported between locations.

The constraints in this model, which are based on attributes of objects and links, may be divided into four categories (Bodin et al., 1983):

1- Constraints based on attributes of objects, like the capacity limit of vehicles.

2- Constraints based on attributes of links, like the consistency between type of goods and vehicles.

3- Constraints based on the consistency of elements of an object, like a constraint on the mixture of two types of goods in distribution.

4- Constraints imposed on the system by the owner of objects like limitation on working hours of drivers. 
Table 1: Sample attributes of vehicle routing objects

\begin{tabular}{|c|c|c|}
\hline No. & Object/Link & Sample attributes \\
\hline 1 & Facility & $\begin{array}{l}\text { Type } \\
\text { Number } \\
\text { Open and close time } \\
\text { Load/Unload equipments } \\
\text { Load/Unload time } \\
\text { Waiting time } \\
\text { Break time } \\
\text { Dependency to other Facilities } \\
\text { Region }\end{array}$ \\
\hline 2 & Carrier & $\begin{array}{l}\text { Capacity } \\
\text { Number } \\
\text { Ownership } \\
\text { Type } \\
\text { Equipments } \\
\text { Dependency to other Carriers } \\
\text { Dependency to other Locations } \\
\text { Delivery Costs } \\
\text { Drivers: } \\
\quad \text { Work start and finish time } \\
\quad \text { Familiarity with regions } \\
\quad \text { Break time } \\
\quad \text { Pay structure } \\
\end{array}$ \\
\hline 3 & Distribution Network & $\begin{array}{l}\text { Road Type } \\
\text { One way roads } \\
\text { U-turn avoidance } \\
\text { Restriction on vehicle (type/capacity) } \\
\text { Toll } \\
\text { Mean vehicle speed }\end{array}$ \\
\hline 4 & Goods & $\begin{array}{l}\text { Type } \\
\text { Quantity } \\
\text { Conflict with other goods } \\
\text { Special carrier requirements } \\
\text { Priority in delivery }\end{array}$ \\
\hline 5 & Load & $\begin{array}{l}\text { Quantity } \\
\text { Split rules } \\
\text { Priority on delivery } \\
\text { Dependency on other loads }\end{array}$ \\
\hline 6 & Trip & $\begin{array}{l}\text { Number of route per vehicle } \\
\text { Number of stops } \\
\text { Region } \\
\text { Dependency on other trips }\end{array}$ \\
\hline 7 & Path & $\begin{array}{l}\text { Road Segment } \\
\text { Distance }\end{array}$ \\
\hline 8 & Demand/Supply & $\begin{array}{l}\text { Type } \\
\text { Source } \\
\text { Destination } \\
\text { Quantity } \\
\text { Uncertainty in quantity } \\
\text { Uncertainty in time } \\
\text { Time window } \\
\text { The time when the demand information become available } \\
\text { Customer preferences } \\
\text { Delivery Type }\end{array}$ \\
\hline
\end{tabular}

Sample attributes of VRSP objects are illustrated in Table 1. Each attribute of an object may be the source of a particular constraint. Using the above framework, various types of problems may be defined.
As the above table shows, despite a simple definition, VRSP is a complex optimization problem. The objective function of such a problem may be defined based on a function of any attribute of the problem solution. The objective function might be maximization, like maximizing capacity utilization, minimization, like minimizing travel 
time, mini-maxing like balancing the load between trucks, or any combination of the above.

\section{CARS modeling and solution approach}

The complex and dynamic environment of businesses calls for flexible systems. A powerful optimization algorithm without the ability to handle specific users' needs is completely ineffective. In real cases, numerous circumstances arise based on a specific situation in one or several objects. For example, if the user wants to avoid assignment of a specific driver to a store, the system should efficiently support it. On one hand, development of system architecture to handle the detail requirements of users results in high complexity of the system. On the other hand, from a practical point of view, the complexity of a system is regarded as a disadvantage.

In developing an optimization system for real problems, the most critical consideration is the complexity and broad range of requirements of clients in the design stage.
Developing a system architecture without taking a particular function into account would either hinder the application of the system or deteriorate its efficiency. The most appropriate solution is to build enough flexibility into the system to adhere with any user requirements. For example, providing a utility to assist users in deciding on some aspects of the solution enables the system to cope with most dynamic business requirements.

The architecture of CARS is developed based on the distribution object models, considering most of variations which may be found in practice. The data structure is the foundation of an optimization system. The flexibility of the system and the configuration of objects to represent the real world problem are based on the data structure. Also, development of data structure according to the logic of the algorithm highly enhances the efficiency of system in solving the problem.



Figure 3: The VRSP objects map used in CARS [30]

The VRSP objects and their rough relationships are shown in Figure 3. This object map is developed by using the VRSP basic model presented in Section 2, and is a foundation for CARS development. These objects are divided into two categories, static and dynamic. Static objects are produced from the problem data, while dynamic objects are created by the system during the construction of a solution to the problem. Static data are related to locations, goods, orders, distribution network and vehicles. Dynamic objects are mainly the components of the problem results. Each object has individual attributes and some relational attributes, which maintain its relation with other objects. Constraints of the problem are formed by using the individual or relational attributes of objects.

The objective function is the weighted sum of several attributes of the solution. The user can set the components of the solution as well as their weights. If cost is the objective, several predefined cost components are available, and the user can select them from a list and set their weights to construct the cost function. In real cases, it is preferred to allow the system to handle soft constraints, which are managerial rather physical constraints. In some cases, such as time window constraint, company policies tolerate some deviation from the target values. The weighted sum of soft constraints is also included in the cost function. An overview of optimization algorithm will be described in the next section.

\section{CARS optimization engine}

VRSP are known to be NP-hard - rather impossible to solve mathematically (Tan, Lee \& Ou, 2001). Thus, efficient heuristic algorithms should be exploited to tackle this class of problems. Several families of heuristics have been developed for VRSP. Heuristics approaches for solving VRSP can be broadly classified into four categories namely, construction, improvement, evolutionary and 
learning approaches (Cordeau et al., 2004). Smart combination of the routines from two or more approaches may also provide promising hybrid algorithms (Tan, Lee \& Ou, 2001; Moscato \& Cotta, 2003). In this section, first the framework of these approaches will be reviewed.

\section{Construction algorithms}

In construction heuristics, nodes are selected successively until a final solution has been built. Saving (Clarke \& Wright, 1964) and Sweep (Gillett \& Miller, 1974) are the most popular algorithms in this class. In sequential implementation one route is constructed in each iteration, while in parallel version several routes are simultaneously built (Laporte et al., 2000). Other well known construction algorithms are "cluster first then route" (Fisher \& Jaikumar, 1981) and "route first then cluster" (Beasley, 1983). In applied algorithms, this type of routines is widely used to construct the initial solution.

\section{Improvement algorithms}

In this type of algorithms, the initial solution is iteratively improved by exploring solution space. Local search algorithms are the most widely used classical improvement algorithms. The structure of local search algorithms can be divided into three components (Ching \& Russel, 1996). The first component is the routine for constructing initial solution. The algorithm may start with a solution constructed randomly or utilize a construction algorithm to obtain a good starting point. Neighborhood structure is another component which heavily influences the behavior of the algorithm. It can be defined by choosing the type of move and the length of string for move (Laporte et al., 2000).

Evaluation rules are other component of local search algorithms. Two extreme strategies are accept-first and accept-best (Bräysy \& Gendreau, 2000). In accept-first strategy the first neighbor which improves current solution will be accepted, while in accept-best strategy the best possible neighbor will be accepted.

In advanced improvement algorithms, which are known as metaheuristics, a sophisticated search routine is exploited to escape the local optima trap and obtain high quality results. Simulated Annealing (SA) (Ching \& Russel, 1996) and Tabu Search (TS) (Branddao \& Mercer, 1997; Glover, 1993; Glover \& Laguna, 1997) are the most well known algorithms in this category. High flexibility and performance of these algorithms make them as the most promising candidates for optimization engine in advanced optimization systems. In our experiences of various practical applications (Modares, Somhom \& Enkawa, 1997; Somhom, Modares \& Enkawa, 1987), SA algorithms need more parameter adjustment and provide lower robustness compared to TS ones.

\section{Evolutionary algorithms}

The basic mechanism in evolutionary approach is combining selected members in a set of generated solutions. Genetic Algorithms (GA) (Homberger \& Gehring, 1999; Thangiah \& NygardJuell, 1991) and Memetic Algorithms (Moscato \& Cotta, 2003) are the most applied heuristics in this class. The foundation of GA is the survival of fitness principle which maintains a high probability of generating the highest level compatible with the environment. Solutions interact, mix together and produce new offspring that hopefully retain good characteristics of the parents. Selection, recombination and mutation are basic operators of GA that conduct evolution process toward higher quality solutions. The most important issue in application of GA to a specific problem is representation phase in which features of the problem are encoded as a chromosome to define a member of the solution population. This condition hinders application of GA to environments in which the specification of problem continually changes according to new situation in business.

\section{Learning algorithms:}

The Neural Network (NN) and Ant Algorithms (Reimann, Doerner \& Hart, 2004) are the most prominent approaches in this category. We have developed several NN algorithms for Traveling Salesman and Vehicle Routing Problems (Modares, Somhom \& Enkawa, 1997; Somhom, Modares \& Enkawa, 1997; Somhom, Modares \& Enkawa, 1999a; Somhom, Modares \& Enkawa, 1999b). In these algorithms, specifications of the optimization problem are embedded into the configuration of network and learning process. Therefore, the algorithm is highly problem specific. Despite their promising results, these algorithms need more enhancements to be incorporated into commercial optimization systems.

\section{CARS' optimization algorithm}

As indicated by Cordeau et al. (2004),"Algorithms are highly accurate and some are also quite fast. What is now needed is greater stress on simplicity and flexibility." Our experience in system development confirms the above statement. In developing CARS algorithm, the most important criteria have been flexibility and speed. Most of new routines developed by researchers are problem specific. They might improve the quality of results at the expense of reducing the flexibility of algorithm to handle new constraints or increasing its computation time. In this section, first, the initial solution and neighborhood generation, which are two pillars of improvement heuristics, are described and then the algorithm is outlined. 
In TS algorithm, during the search process, current solution may deteriorate from one iteration to the next. To avoid cycling, recent explored solutions are temporarily declared forbidden by putting their selected attributes in the Tabu list. The TS algorithm has been evolved over time and several innovative features are included in this algorithm by researchers to enhance its performance (Bräysy \& Gendreau, 2000). Enhanced neighborhood generation mechanism, using various diversification and intensification strategies to guide the search, post optimization, combination with other algorithms, and parallel implementation are various strategies which are considered by researchers. The granular Tabu search algorithm of Toth and Vigo (2003), the unified Tabu search algorithm of Cordeau, Laporte \& Mercier (2001); Cordeau, Laporte \& Mercier, (2004) and Taillard et al. (1997) algorithm are recent algorithms which have shown promising results.

While we have considered innovative features of proposed algorithm by researchers, CARS' TS algorithm has unique features, described below. TS algorithms start with an initial solution which can be developed by a simple construction algorithm. Solomon (Somhom et al., 1987) proposed several heuristics for the VRSP which are suitable candidates for building initial solution of TS algorithms. Among them, I1 algorithm which is a cheapest insertion routine has been used by several researchers for building initial solutions. We have implemented a modified version of Solomon's I1 algorithm for general VRSP.

The algorithm starts with initializing a route using a seed customer. The seed customer is the customer with the lowest cost when assigned. The remaining unassigned customers are sequentially inserted into this route as far as capacity restriction of truck or other hard constraints permits. For inserting a customer in a route, total cost of insertion is evaluated. The initialization and insertion procedure continue until all customers are serviced.

The most popular neighbor generation operators are Relocate, Exchange, 2-opt* and CROSS [5]. The Relocate operator moves one visit from its position into a new position, while the Exchange operator swaps two visits. In order to improve the quality of solution or speed up the algorithm, numerous enhanced routines have been proposed by researchers (Bräysy \& Gendreau, 2000; Laporte et al., 2000). $\lambda$-interchange, GENI-Exchange (Gendreau, Hertz \& Laporte, 1992), eject chain (Glover, 1991) and cyclic transfer (Thompsn \& Psaraftis, 1993) are other successful routines.

Our experiments show that a combination of relocation, exchange and 2opt* can provide high quality results within low computational time. We have examined more complex routines like CROSS and 3-opt, which resulted in no meaningful additional value and in higher computation time. Since the problem contains contradicting objectives, using specific strategies (Garcia, Potvin \& Rousseau, 1994; Lau, Sim \& Teo, 2003) to artificially help the algorithm in finding better solutions, is harmful and reduces the effectiveness of the algorithm.

In implementing the algorithm for real problems, managerial restrictions are considered as soft constraints. For each soft constraint, a penalty term will appear in the objective function. In CARS only basic constraints like capacity restriction or route length limit are considered as a hard constraint. By increasing the weight of penalty term for a given soft constraint in the objective function, the possibility of satisfying this constraint will increase.

The algorithms starts by generating an initial solution based on the procedure explained earlier. The neighborhood generating mechanisms, namely Relocate, Exchange and 2 opt* $^{*}$ are selected randomly. Based on several experiments, the chance of selecting 2opt* operator is set to be five times less than Relocate and Exchange. Since the associated cost of each stop, trip and tour is maintained separately, the search procedure is guided by considering attractive moves. This is a specific implementation and extended application of candidate-list strategy (Clarke \& Wright, 1964), which has been applied in several other algorithms (Toth \& Vigo, 2003; Johnson \& McGeoch, 1997). This innovative strategy considerably speeds up the search process and lead to high quality results.

When customer $i$ in route $k$ is moved, its reinsertion is forbidden for next $\theta$ iteration by keeping (i.k) attributes in Tabu list. Through an aspiration criterion, neighbor solutions with lower cost than the best found solution are permitted to be accepted even if their attributes are in the Tabu list. In generating neighbor solution hard constraints are also controlled. It has been shown by researchers (Taillard et al., 1997) that a dynamic Tabu list size tends to give better results than fixed one. The parameter $\theta$ which indicates the size of Tabu list is a randomly generated number between $\theta_{\max }$ and $\theta_{\min }$. We set $\theta_{\max }$ to 10 and $\theta_{\min }$ to 5 similar to other researchers $[19,45]$. The algorithms stop after reaching either the time set by the user or maximum allowed iterations.

\section{Experiment results}

In order to evaluate the performance of CARS optimization engine, a comprehensive experiment is conducted on a set of standard problem available in the literature. In this experiment we have used the Christofides, Mingozzi and Toth (CMT) 14 standard VRSP benchmark instances (Christofides, Mingozzi \& Toth, 1979). These problems contain 50 to 199 cities in addition to depot. Problems marked as $\mathrm{C}$ type have capacity restriction and type $\mathrm{D}$ problems have route length constraint. Our intention was to demonstrate the capability of CARS' optimization engine on classical VRSP for which enormous research and experiments has been done. The performance of CARS is compared with several best known advanced heuristic algorithms, namely, Taburoute (Gendreau, Hertz \& Laporte, 1994), Taillard TS (Taillard, 1993), Berger and 
Barkaoui algorithm (2004), Granular TS (Toth \& Vigo, 2003) and Unified TS (Cordeau, 2001).

Table 2 demonstrated the results of CARS in comparison with the selected algorithms. The reported results for CARS are the best found solutions over 5 runs. As this table shows, the average deviation of CARS results from the best known solutions is 0.55 percent. In three instances, CARS provides the best known solutions. This experiment shows that CARS can provide comparable results with sophisticated TS algorithm for classical VRSP. Since CARS is designed for real complex problems, we believe that it can easily attain higher quality solutions than algorithms which are designed and tested for standard problems. Most of available algorithms are quite specific and need special modification for more complex cases.

The computation time of algorithms cannot be compared directly, since they have run on different machines. CARS average run time for CMT set is 0.5 minutes, which is also an outstanding performance. This experiments shows that CARS can provide high quality results comparing to the leading edge heuristic algorithms. Another important feature of CARS is simplicity. Although, most of algorithms have specific parameters to be adjusted by users, CARS has no optimization parameter.

In order to demonstrate the performance of CARS in solving real problems, from recent practical applications, some actual results are provided here. The performance is compared to other methodologies which can handle comparable practical problems. Table 2 illustrates the results of improvements achieved by CARS for two companies in food industries. The number of trucks for each company is around 150 and 250.

Table 2: Comparison of CARS' results for the selected algorithms on CMT benchmark instances

\begin{tabular}{|c|c|c|c|c|c|c|c|c|c|c|c|c|c|c|}
\hline \multirow{2}{*}{ No. } & \multirow{2}{*}{ Size } & \multirow{2}{*}{ Type } & \multicolumn{2}{|c|}{ Taburoute $^{1}$} & \multirow{2}{*}{$\frac{\text { Taillard }^{2}}{\text { Value }}$} & \multicolumn{2}{|c|}{ 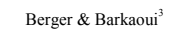 } & \multicolumn{2}{|c|}{ Granular TS } & \multicolumn{2}{|c|}{ Unified $\mathrm{TS}^{5}$} & \multicolumn{2}{|c|}{ CARS } & \multirow{2}{*}{ Best Known Solution } \\
\hline & & & Value & Minutes $^{6}$ & & Value & Minutes ${ }^{7}$ & Value & Minutes $^{8}$ & Value & Minutes $^{9}$ & Value & Minutes $^{10}$ & \\
\hline 1 & 50 & C & 524.61 & 6.00 & 524.61 & 524.61 & 2.00 & 524.61 & 0.81 & 524.61 & 4.57 & 524.61 & 0.11 & 524.61 \\
\hline 2 & 75 & $\mathrm{C}$ & 835.77 & 53.80 & 835.26 & 835.26 & 14.33 & 838.6 & 2.21 & 835.45 & 7.27 & 839.61 & 0.31 & 835.26 \\
\hline 3 & 100 & C & 829.45 & 18.40 & 826.14 & 827.39 & 27.90 & 828.56 & 2.39 & 829.44 & 11.23 & 829.18 & 0.22 & 826.14 \\
\hline 4 & 150 & $\mathrm{C}$ & 1036.16 & 58.80 & 1028.42 & 1036.16 & 48.98 & 1033.21 & 4.51 & 1038.44 & 18.72 & 1033.21 & 0.45 & 1028.42 \\
\hline 5 & 199 & C & 1322.65 & 90.90 & 1298.79 & 1324.06 & 55.41 & 1318.25 & 7.50 & 1305.87 & 28.10 & 1298.79 & 0.70 & 1291.45 \\
\hline 6 & 50 & $\mathrm{C}, \mathrm{D}$ & 555.43 & 13.50 & 555.43 & 555.43 & 2.33 & 555.43 & 0.86 & 555.43 & 4.61 & 555.43 & 0.20 & 555.43 \\
\hline 7 & 75 & $\mathrm{C}, \mathrm{D}$ & 913.23 & 54.60 & 909.68 & 909.68 & 10.50 & 920.72 & 2.75 & 909.68 & 7.55 & 913.33 & 0.53 & 909.68 \\
\hline 8 & 100 & $\mathrm{C}, \mathrm{D}$ & 865.94 & 25.60 & 865.94 & 868.32 & 5.05 & 869.48 & 2.90 & 866.38 & 11.17 & 869.48 & 0.30 & 865.94 \\
\hline 9 & 150 & $\mathrm{C}, \mathrm{D}$ & 1177.76 & 71.00 & 1162.55 & 1169.15 & 17.88 & 1173.12 & 5.67 & 1171.81 & 19.17 & 1176.50 & 0.83 & 1162.55 \\
\hline 10 & 199 & $\mathrm{C}, \mathrm{D}$ & 1418.51 & 99.80 & 1397.94 & 1418.79 & 43.86 & 1435.74 & 9.11 & 1415.4 & 29.74 & 1397.94 & 0.90 & 1395.85 \\
\hline 11 & 120 & C & 1073.47 & 22.20 & 1042.11 & 1043.11 & 22.43 & 1042.87 & 3.18 & 1074.13 & 14.15 & 1074.13 & 0.63 & 1042.11 \\
\hline 12 & 100 & C & 819.56 & 16.00 & 819.56 & 819.56 & 7.21 & 819.56 & 1.10 & 819.56 & 10.99 & 819.56 & 0.57 & 819.56 \\
\hline 13 & 120 & $\mathrm{C}, \mathrm{D}$ & 1573.81 & 59.20 & 1541.14 & 1553.12 & 34.91 & 1545.51 & 9.34 & 1568.91 & 14.53 & 1545.98 & 0.77 & 1541.14 \\
\hline 14 & 100 & $\mathrm{C}, \mathrm{D}$ & 866.37 & 65.70 & 866.37 & 866.37 & 4.73 & 866.37 & 1.41 & 866.53 & 10.65 & 868.50 & 0.53 & 866.37 \\
\hline \multicolumn{3}{|c|}{ Percent Deviation from the best known } & $0.86 \%$ & & $0.06 \%$ & $0.48 \%$ & & $0.69 \%$ & & $0.69 \%$ & & $0.55 \%$ & & \\
\hline \multicolumn{8}{|c|}{$\begin{array}{l}\text { ' Gendreau et al ., } 1994 \\
{ }^{2} \text { Taillard (1993) (Time is not available) } \\
{ }^{3} \text { Berger \& Barkaoui (2004) } \\
\text { Toth \& Vigo (2003) } \\
\text { 5 Cordea et al.2001 }\end{array}$} & \multicolumn{7}{|c|}{$\begin{array}{l}{ }^{6} \text { Silicon Graphics workstation } 5.7 \\
{ }^{7} \text { Pentium } 400 \mathrm{MHz} \text { PC } \\
{ }^{8} \text { Pentium } 200 \mathrm{MHz} \text { PC } \\
\left.{ }^{9} \text { Sun Ultrasparc } 10 \text { ( } 440 \mathrm{MHz}\right) .\end{array}$} \\
\hline
\end{tabular}

To demonstrate the effectiveness of the system, a sample of ten consecutive days planning data is obtained from two companies who are willing to utilize CARS as their new logistics planning system. The results attained by CARS are compared with those of their current system in use.

The first company was using an advanced planning and scheduling system. The second company was using an uncomplicated planning system with an optimization engine which can interact with planners and let them improve the results based on their knowledge.

To evaluate the system performance, three measurement indices are considered. Distance, which is a traditional index in the evaluation of VRSP, is the primary index. The most important index in practice is distribution cost. This cost is comprised of distribution mileage cost, fixed cost of utilized trucks and overtime cost. The customer service is 
another important index in practice. This index is calculated by multiplying time window satisfaction and customer priority.

Table 3 shows the percent of improvement in results of distribution, compared to previous systems, in two sample companies over ten days. The results indicate that CARS can substantially improve the effectiveness of distribution planning in both cases. Although it is not a solid statistical proof, it provides an initial indication of the value of our system in practice.

The system in Company A, compared with Company B, can provide better results in distance and cost minimization in the expense of lower service level. Since in Company B the results are refined by experts, they can easily improve the customer service level. However, this increases distance and cost. Overall, it seems that the optimization engine of CARS provides much better compromise between different objectives and higher solution quality.

Table 3: Percent of Improvement in performance using CARS in sample

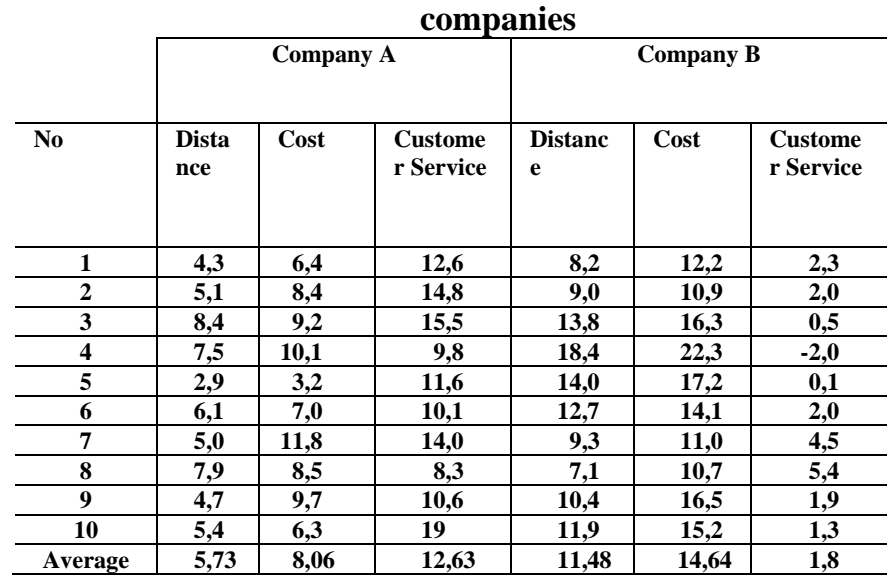

\section{CARS features}

The complexity of VRSP requires a sophisticated planning and evaluation system. It is very difficult for a dispatcher to evaluate and possibly modify the results provided by the system, just by examining the sequence of events. The system should help him/her to consider the results from several dimensions. To meet this requirement, several viewing tool are embedded in CARS. Users can browse the final results in tabular, Gantt chart, tree and map formats.

The tabular view presents the sequence of visits by vehicles, in a table along with detail and aggregate information of the result. This view provides the user with an overview of the plan in order to efficiently evaluate the distribution of load between trucks, operation time and capacity usage.
The Gantt chart view maps the results based on time and load of trucks. This view shows the schedule of each truck, along with its utilized capacity in any stage of service. Travel time, load and unload times, rest and return periods are presented with different colours.

The map view assists the user to follow geographical movement of vehicles, and to focus on a particular arrangement of routes and vehicles on the map. This view provides dispatchers with quick placement of vehicles on the map and helps them to modify the results according to their know-how. In practice, most of modifications and evaluations are performed on this view, in a convenient visual manner.

In tree view, the solution is organized in a convenient tree format. Each branch, which represents a vehicle, can be expanded to exhibits trips, routes and stops. The important attributes of each object along with statistical charts, which provide visual comparative analysis of the selected attributes of the object, are shown in a linked window. This intuitive view enhances the efficiency of modification. In all of the above views, a modification can be performed by just using drag and drop. Figure 4 illustrates a snapshot of several views of CARS.

By using the 'cost components tool', one may evaluate system performance, resource utilization and customer service level, which are measured by several indices. This tool can demonstrates the outcome of intended modifications and assist the user to find the best improvement alternative.

Due to the nature of soft constraints in the system, cases may be encountered in which some of soft constraints are not satisfied. A soft constraint evaluation tool is incorporated in CARS to notify users of the status of soft constraints in the plan. In the initial setting of the system, depending on the degree of violation, several zones for each soft constraint may be set and a color can be assigned to each zone. In all of views, the violated soft constraints will be marked by appropriate color. This simple tool facilitates fast detection of critical cases in the plan.

Since several aspects of an actual problem are not quantifiable, the user may need to override the solution provided by the system, in some cases. CARS provides the possibility to utilize the power of algorithm in modification procedure. For example, if the user wants to remove a stop from its position, the system immediately guides him to the best position where it might be inserted. The system can search for the best position, in the solution, in a trip or in a route. In the same way, the whole load of a truck can be transferred to the best available positions to take the truck out of service. 


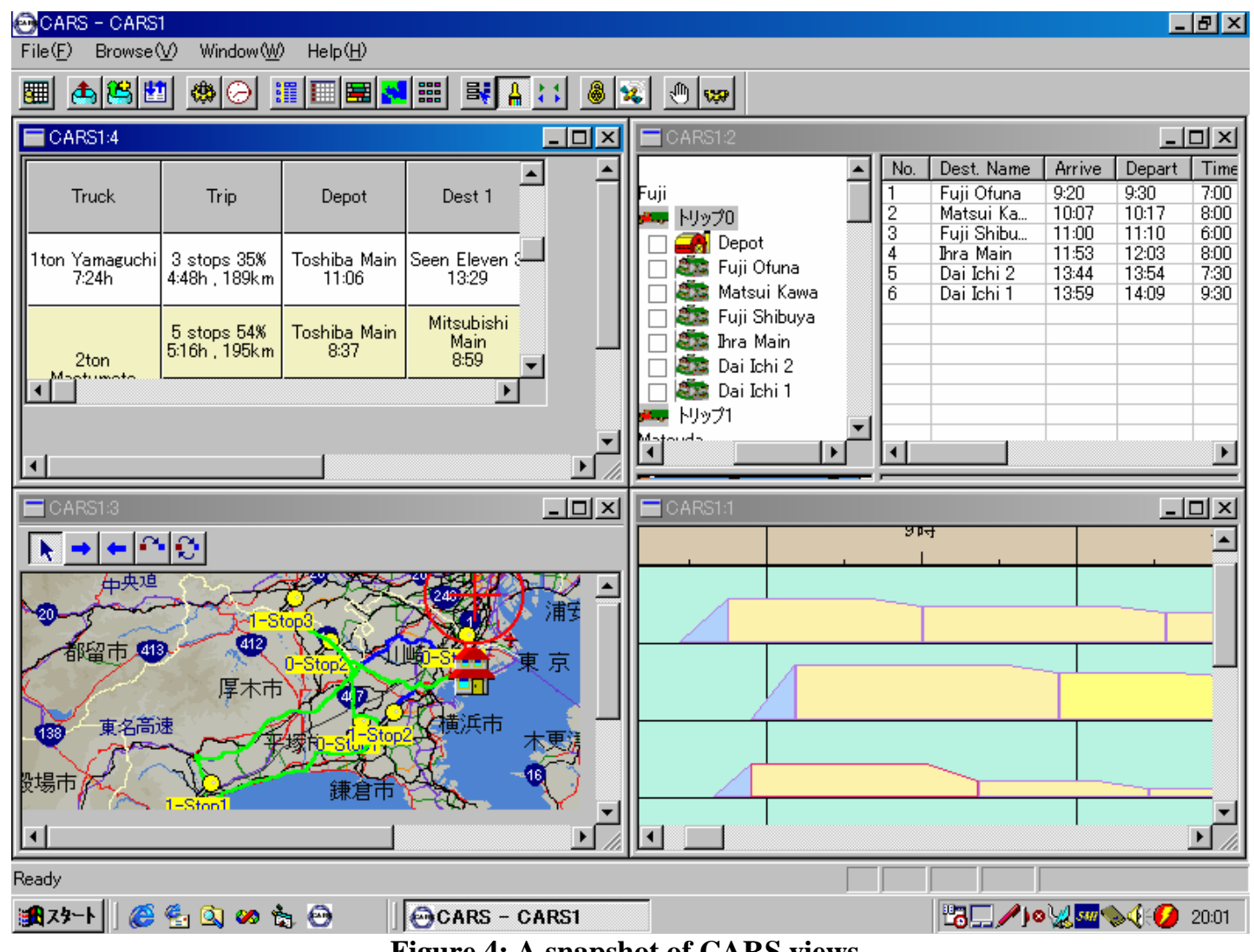

Figure 4: A snapshot of CARS views.

Geographic Information System (GIS) is an essential part of the system, since the distribution network data is the basis for the schedule and cost calculations. It also facilitates the accurate address registration by marking the location of sites on the map. CARS calculates distance or travel time between locations in offline mode and saves it for future use. This data can be improved by using daily driver reports or using Geographic Positioning System (GPS) to track vehicles and record actual travel time. This feature enables the system to improve the accuracy of plan by lowering the discrepancy between planned and actual data.

It is worthy to note that CARS has the potential to be used for Strategic Distribution Planning issues such as territory planning and location analysis. Similarly, the configuration of a supply chain may be analyzed by virtual generation of candidate site and based on actual data or assessment of distribution and site costs.

\section{Conclusion}

This paper described the modeling and design approach as well as main features of an advanced optimization system for various types of VRSP. CARS system can be used within an integrated supply chain planning system to optimize distribution operations. It can handle various logistics configurations and may be applied to any industry with minor customization. The system is uniquely developed to handle a large variety of objects and configurations in distribution planning problems.

The main contribution of CARS is using advanced heuristics to tackle such a complex problem and practically improve the efficiency of distribution planning systems. It uses a specialized optimization engine whose underlying structure is based on the proposed classification. CARS is a flexible system able to solve VRSP with complex constraints by its powerful optimization algorithms. It provides the user with advanced functionalities for analyzing the results, modifying the problem instances and evaluating alternative solutions.

CARS, as with most available algorithms, assumes that the travel times between locations are constant throughout the day. In the real-world, the travel times are time dependent and differ according to the traffic load. In the future research, parallel implementation of the algorithm is another development which is necessary to speedup the solution process. Further work should be aimed at enhancement of the algorithm to address the above issues. These developments will enable the system to match precise requirements of e-commerce fulfillment and home delivery problems.

\section{References}

Beasley, J.E. 1983. 'Route-first cluster-second methods for vehicle routing', Omeg, 11: 403-408. 
Berger J. \& Barkaoui, M. 2004. 'A new hybrid genetic algorithm for the capacitated vehicle routing problem', Journal of the Operational Research Society, 54:12541262.

Bodin L., Golden B., Assad, A. \& Ball, M. 1983. 'Routing and scheduling of vehicles and crews: The state of the art', Computer and Operations Research, 10: 63-211.

Branddao, J. \& Mercer, A. 1997. 'A Tabu search algorithm for the multi-depot vehicle routing and scheduling problem', European Journal of Operational Research, 100:180-191.

Bräysy, O. \& Gendreau, M. 2000. 'Vehicle routing problem with time windows, Part II: Metaheuristics', Transportation Science, 39:119-139.

Ching, W. \& Russel, R. 1996. 'Simulated annealing metaheuristics for the vehicle routing with time window', Annals of Operations Research, 63:3-27.

Chopra, S., Dougan, D. \& Taylor, G. 2001. 'B2B 2commerce opportunities,' Supply Chain Management Review, May/June:50-58.

Chopra, S. \& Van Miegham, J.A., 2000. 'Which e-business is right for your supply chain?' Supply Chain Management Review, July/August:32-40.

Chopra, S. \& Meindl, P. 2004. Supply chain management: Strategy, planning, operation. Prentice Hall.

Christofides, N., Mingozzi, A. \& Toth, P. 1979. 'The vehicle routing problem.' In Christofides, N., Mingozzi, A., Toth, P. \& Sandi, C. (Eds.). Combinatorial optimization. Chichester: Wiley.

Clarke, G. \& Wright, J.W. 1964. 'Scheduling of vehicles from a central depot to a number of delivery points', Operations Research, 12:568-581.

Cordeau, J.-F., Laporte, G. \& Mercier, A. 2001. 'A unified tabu search heuristic for vehicle routing problems with time windows', Journal of the Operational Research Society, 52: 928-936.

Cordeau J.-F., Laporte G., \& Mercier A. 2004. 'An Improved tabu search algorithm for the handling of route duration constraints in vehicle routing problems with time windows', Journal of the Operational Research Society, 55:542-546.

Cordeau, J.-F., Gendreau, M., Hertz, A., Laporte, G. \& Sormany, J.-S. 2004. 'New heuristics for the vehicle routing problem'. Working Paper Les Cahiers du GERAD.

Derochers, M., Lenstra, J. \& Savelsberh, M. 1990. 'A classification scheme for vehicle routing and scheduling problem', European Journal of Operational Research, 46: 322-332.
Fisher, M.L. \& Jaikumar, R. 1981. 'A generalized assignment heuristic for the vehicle routing problem', Networks, 11:109-124.

Garcia, B.-L., Potvin, J.-Y. \& Rousseau, J.-M. 1994. 'A parallel implementation of the Tabu search heuristic for vehicle routing problems with time window constraints', Computers and Operations Research 21: 1025-1033.

Gendreau, M., Hertz, A. \& Laporte, G. 1994. ' A Tabu search heuristic for the vehicle routing problem', Management Science, 40:1276-1290.

Gendreau, M., Hertz, A. \& Laporte, G. 1992. 'A new insertion and post-optimization procedures for the traveling salesman problem', Operations Research, 40:1086-1093.

Gillett, B.E. \& Miller, L.R 1974. 'A heuristic algorithm for the vehicle dispatch problem', Operations Research, 22:340-349.

Glover, F. 1991. 'Multilevel Tabu search and embedded search neighborhoods for the traveling salesman problem', Working Paper, College of Business and Administration, University of Colorado.

Glover, F. 1993. 'A user's guide to Tabu search', Annals of Operations Research, 41: 3-28.

Glover, F. \& Laguna, M. 1997. Tabu search. Boston: Kluwer Academic Publishers.

Homberger, J. \& Gehring, H. 1999. 'Two evolutionary metaheuristics for the vehicle routing problem with time windows', Information Systems Operations Reseach, 37:297-318.

Johnson, D. S. \& McGeoch, L. A. 1997. 'The traveling salesman problem: A case study'. In Aarts, E. H. \& Lenstra, J. K. (Eds.). Local search in combinatorial optimization. Chichester : Wiley.

Laporte, G., Gendreau, M., Potvin, J. \& Semet, F. 2000. 'Classical and modern heuristics for the vehicle routing', International Transactions in Operational Research, 7: 285-300

Lau, H. C., Sim, M. \& Teo, K. M. 2003. 'Vehicle routing problem with time windows and a limited number of vehicles', European Journal of Operational Research, 148: 559-568.

Modares, A., Somhom, S. \& Enkawa, T. 1997. 'A competitive neural network algorithm for solving vehicle routing problems', International Journal on Computer and Industrial Engineering, 33: 473-476.

Moscato, P. \& Cotta, C. 2003. 'A gentle introduction to memetic algorithms'. In Glover, F. \& Kochenberger, G.A. (Eds.). Handbook of metaheuristics, p.p.105-144. Boston: Kluwer. 
Reimann, M., Doerner, K. \& Hart, R.F. 2004. 'D-ants: Savings based ants divide and conquer the vehicle routing problem', Computers \& Operations Research, 31: 563-591.

Osman, I. 1993. 'Metastrategy simulated annealing and Tabu search algorithms for the vehicle routing problems', Annals of Operations Research , 41: 421-452.

Potvin, J. Y. \& Rousseau, J. M. 1995. 'An exchange heuristic for routing problems with time windows', Journal of Operational Research Society, 46:1433-1446.

Somhom, S., Modares, A. \& Enkawa, T. 1987. 'Algorithms for the vehicle routing and scheduling problems with time window constraints', Operations Research, 35: 254-265.

Somhom, S., Modares, A. \& Enkawa, T. 1997. 'A selforganizing neural network model for the traveling salesman problem', Journal of the Operational Research Society, 48: 919-928.

Somhom, S., Modares, A. \& Enkawa, T. 1999a. 'Competition-based neural network for the multiple travelling salesman problem with minimax objective', International Journal of Computers and Operations Research, 26: 395-407.

Somhom, S. Modares, A. \& Enkawa, T. 1999b. 'Selforganizing neural network approach for multiple traveling salesman and vehicle routing problems', International Transactions in Operational Research, 6:591-606.

Taillard, E. 1993. 'Parallel iterative search methods for vehicle routing problems', Networks, 23: 661-673.

Taillard, E., Badeau, P., Gendreau, M., Guertin, F. \& Potvin J.-Y. 1997. 'A Tabu search heuristic for the vehicle routing problem with soft time windows', Transportation Science, 31:170-186.

Tan, K. C., Lee, L. H. \& Ou, K. 2001. 'Artificial intelligence heuristics in solving vehicle routing problems with time window constraints', Artificial Intelligence, 14: 825-837.

Thangiah, S.R., Nygard K.E. \& Juell, P.L. 1991. 'GIDEON: A genetic algorithm system for vehicle routing with time windows', Proceedings of the 7th IEEE Conference. Artificial Intelligence Applications. Los Alamitos: IEEE Computer Society Press, p.p.322-328

Thangiah, S. R., Osman, I. \& Sun, T. 1994. ' Hybrid genetic algorithm, simulated annealing and Tabu search methods for vehicle routing problems with time windows.' Working Paper UKC/IMS/OR94/4, Institute of Mathematics and Statistics, University of Kent, Canterbury. UK.

Thompson, P. M. \& Psaraftis, H. N. 1993. 'Cyclic transfer algorithms for multi-vehicle routing and scheduling problems', Operations Research, 41: 935-946.
Toth, P. \& Vigo, D. 2003. 'The granular Tabu search and its application to the vehicle routing problem', INFORMS Journal on Computing, 15: 333-346. 